\title{
A Comparative Analysis of the Fitness of Collegiate Dancers as compared to Collegiate Volleyball and Softball Players
}

\author{
Leanna Miller* \& Fred L. Miller III \\ Department of Kinesiology, Anderson University, Anderson, IN \\ Student:Imiller@anderson.edu* \\ Mentor:fmiller@huntington.edu
}

\begin{abstract}
Few studies directly compare the various fitness components of collegiate dancers and athletes in acknowledged sports. The limited studies that do exist fail to consider certain variables or to assess multiple fitness components. The purpose of this study was to provide a more comprehensive comparison of collegiate dancers and collegiate volleyball and softball players. We used multiple fitness components as an indicator of the comparative physical demands of these activities, to assess whether the physical demands of collegiate dance may warrant a comparable level of medical support as afforded to the volleyball and softball players. Thirty dancers and thirty volleyball and softball players were tested using six different measurements of physical fitness commonly used in athletic assessments at universities. A two-tailed independent $t$-test was performed to determine differences between dancers and athletes in each fitness component. Three tests revealed significant differences between the groups, with the dancers having a lower percent body fat and greater flexibility, and the athletes having greater relative upper body strength. No significant differences were found in relative leg strength, core strength, or estimated $\mathrm{VO}_{2 \max }$. The results of the current study suggest that dance provides training adaptations equivalent to traditional intermittent sports and thus support the use of similar levels of medical care for collegiate dancers to that afforded to collegiate volleyball and softball players.
\end{abstract}

\section{KEYWORDS}

Physical Fitness; Dance; Dance Medicine; Athletics; Dance Health Benefits

\section{INTRODUCTION}

According to statistics provided by the National Dance Education Organization, there are approximately 665 postsecondary institutions offering dance major and minor programs in the United States. There is a recent trend to use dance to achieve fitness. Dance causes training adaptations, but to what extent does a collegiate level, semi-professional dancer experience training adaptations in comparison to a collegiate athlete? There are numerous studies that show dance to be an effective fitness modality, as it improves muscular strength and endurance, cardiorespiratory health, flexibility, and body composition in an enjoyable and creative manner. 1, 2, 3, 4 These studies focus on the fitness benefits of dance training among at-risk adolescents and previously sedentary women, and they do not provide meaningful insight into the benefits and demands of collegiate level dance. Other studies have focused on a demographic and activity level more appropriate for analyzing collegiate dance, but are incomplete or limited in many aspects.

Several previous studies contrasted the fitness gains between genres of dance, suggesting that the athletic demands of dance can differ, but participation in many styles of dance can result in good aerobic fitness. ${ }^{1,2}$ Other studies confirmed that fitness levels vary amongst dancers of different styles. Studies that tested the aerobic fitness of professional contemporary and modern dancers compared to that of professional ballet dancers showed the contemporary and modern dancers to have higher maximal oxygen uptake, lower blood pressure, resting heart rates, and peak heart rate than ballet dancers. ${ }^{2,5}$ The researchers hypothesized that the difference between genres is largely due to the tendency for modern dance numbers to be lengthier than ballet numbers, as well as to the decreased stigma against cross-training for modern dancers in comparison to ballet dancers. ${ }^{5,6}$ Other studies examined muscular strength differences between dancers of different genres. Ballet dancers did not show muscular strength significantly above average fit individuals, but modern dancers had muscular strength similar to that of other traditional athletes. The ballet dancers had lower BMIs than many traditional athletes however, and showed greater flexibility. ${ }^{7}$ Similar studies found that some muscle groups display similar levels of strength in dancers as in athletes, but other muscular strength measurements can be more comparable to those of sedentary individuals, despite the dancers participating in more than five times the amount of physical activity as the non-dancer sample group. 8,9 This may be related to the common practice of dancers to avoid cross-training for fear that cross-training would limit dance ability. 5,8 This lack of comprehensive exercise prompted some professional dancers to have similar results on certain fitness components to sedentary individuals. With proper cross-training, not only did overall fitness of the participating dancers improve, but also the number of injuries was reduced. ${ }^{7}$ These results suggest that the most physically fit dancers are trained in several styles, as well as cross-train to correct muscle imbalances. ${ }^{7,8}$ 
Other discussions addressed the limitations of muscular performance due to the aesthetic nature of dance. One such study suggested the variability in muscle strength was partially due to the importance of aesthetic in dance. The focus of the dancer is not maximal force production, but rather on controlling the force to obtain a certain aesthetic. ${ }^{2,8}$ Researchers suggested that improved muscular strength and endurance also improve coordination, which will yield aesthetic performance improvements. However, there is little research investigating the correlation between increased fitness parameters and aesthetic performance. ${ }^{10}$ Measurements of aesthetic competence are less clearly defined as measurements of fitness, confounding research in aesthetic improvements associated with athletic gain. Further research is needed to find relationships between fitness components and aesthetic performance. If a correlation between a specific fitness component and improved aesthetic performance could be found, dancers could undergo supplementary conditioning focused on that particular fitness component. The same principles used by sports scientists to identify the component of fitness that best improves athletes of a specific sport can be applied to analyze dance performance, with slight modifications to the scales used to analyze performance.

The purpose of this study was to provide a direct comparison of collegiate dancers to traditional collegiate athletes to identify the similarities and differences in the athletic demand of their sports. This was accomplished by comparing the five health-related fitness components of female collegiate dancers and conventional female collegiate athletes, utilizing standard tests commonly used in fitness assessments for collegiate athletes. The direct comparison of body composition, muscular endurance, muscular strength, flexibility, and cardiorespiratory endurance provides insight into the physical demands of dance compared to the physical demands of traditional sports.

\section{METHOD}

\section{Participants}

The participants were college-age females $(\mathrm{n}=60)$ selected from a Midwestern university. Thirty dance majors and thirty traditional athletes in softball or volleyball were tested. The dancers trained for a minimum of five hours a week. They trained in three primary dance techniques - modern, ballet, and jazz - regularly to ensure that they exhibited the fitness gains from multiple styles. The traditional athletes had an active status on either the varsity volleyball or the varsity softball team. At the time of testing, the volleyball players were in-season, and the softball players were nearing the end of preseason training.

\section{Procedures and Instruments of Analysis}

The study compared the fitness of 30 female collegiate dancers to that of 30 traditional collegiate female athletes involved in softball or volleyball. The study included measurements of body composition, cardiovascular endurance, flexibility, muscular endurance, and muscular strength.

By using athletes whose sports have similar aerobic demands to those of dance, it was hoped that the variability of aerobic capacity would be reduced. Muscle strength tests encompassed several muscle groups to provide a more accurate overall assessment of strength. It was hypothesized that the sample group of dancers would display similar fitness levels to the sample group of traditional athletes, supporting the argument for dance to be considered as a sport.

The study was approved by the Human Research Participants Committee at Anderson University. All participants completed a health history form, completed a PAR-Q, and signed an informed consent form prior to testing. Each participant's height and weight was measured prior to testing.

The first measurement was body composition, utilizing a bioelectrical impedance analysis (BIA). The machine was tared, then the participant's sex, weight, and height were entered. Using both hands, the participant held the device fully extended in front of her body. The results of the test were recorded in a table. Each participant was measured with the same device, and the device was calibrated after each use.

The second portion of the test measured flexibility using a standard sit and reach test. The participant removed her shoes and sat on the floor with her legs stretched out in front of her. The sit and reach box was placed so that the participant's feet are at the 26 $\mathrm{cm}$ mark. Keeping knees straight and feet flat against the front end of the test box, the participant leaned forward and pushed her hand up the ruler as far as possible. The test was performed three times. The results were averaged and recorded.

Next the participant performed two tests of muscular strength and one of muscular endurance. Each muscular strength test assessed a different area of the body.

Lower body muscular strength was measured by means of a maximal leg press using the leg press machine. The leg press machine was chosen to provide a more accurate test of actual strength and not training level. To use free weights would have favored any 
participant who was familiar with weight lifting techniques. To utilize the leg press machine, the participant sat with her knees bent and both feet flat against the base panel. The participant straightened her knees against the resistance weight until legs were fully extended, then slowly bent her knees to return to the starting position. The initial weight for leg press was set at the closest machine setting to half of the participant's body weight. Weight was added until the participant had reached the maximum resistance that she could press. To account for differences in body size, the maximal weight pressed was divided by the participant's body weight, and the results were recorded.

Upper body strength was measured by performing an inclined chest press on the hammer press machine. The participant began seated at the machine, using proper form and posture. The participant grasped the handles and extended her arms forward and up. The initial weight for the chest press was set at the closest machine setting to one fifth of the participant's body weight. Weight was added until the participant could not lift the load. Like the first test of muscular strength, the maximal weight was divided by the participant's body weight to obtain a measurement of relative strength. Results were recorded.

The participant performed a test of core endurance by performing a standard curl up test. The participant lay in a supine position on a mat, with her feet flat on the floor and her knees bent at a 90-degree angle. The arms were extended to sides with fingers touching a piece of masking tape. A metronome was set at 40 beats per minute. At the first sound, the participant lifted her torso off the floor until her fingertips reach a second piece of tape placed $12 \mathrm{~cm}$ past the first piece of tape. At the second sound, the participant lowered back to starting position. This was repeated in time until the participant felt she could not continue or broke pace with the metronome. The number of completed curl ups was recorded. The maximum number of curl ups allowed was one hundred curl ups.

Finally, cardiorespiratory endurance was measured by a one-mile jog test. The participant completed one warm up lap at a moderate walk. Then the participant completed a mile at a comfortable steady jogging pace, with a total time greater than nine minutes. According to the testing protocol, heart rate at the end of the mile should not exceed 180 bpm. The participant's mile time was recorded. Heart rate was measured and recorded. An estimate of $\mathrm{VO}_{2 \max }$ was calculated using the following equation:

$$
\mathrm{VO}_{2 \max }(\mathrm{ml} / \mathrm{kg} / \mathrm{min}) \approx 100.5-(0.1636 \times w)-(1.438 \times t)-(0.1928 \times H R), \quad \text { Equation } 1
$$

where $w$ denotes body weight in $\mathrm{kg}, t$ is time in minutes and $H R$ is post-exercise heart rate in bpm. Every participant was tested on the same indoor track.

\section{Data Analysis}

Results from each test were entered into Microsoft Excel. The mean, range, and standard deviation for each test were calculated for the dancer sample and for the traditional athlete sample. The results for each sample group were compared using a two-tailed independent $t$-test to determine if there was a significant difference $(\alpha=0.05)$ between the means of the two sample groups.

\section{RESULTS}

The sample group of dancers $(\mathrm{N}=30)$ and the sample group of traditional athletes $(\mathrm{N}=30)$ completed six different fitness tests.

\begin{tabular}{|c|c|c|c|c|}
\hline Variable & $\begin{array}{c}\text { Dancer Mean } \pm \text { Standard } \\
\text { Deviation } \\
\text { (Range) }\end{array}$ & $\begin{array}{c}\text { Athlete Mean } \pm \text { Standard } \\
\text { Deviation } \\
\text { (Range) }\end{array}$ & $\begin{array}{c}t \text {-test } \\
\text { value } \\
t(58)=\end{array}$ & $P$ value \\
\hline Height (in) & $65.33 \pm 2.63(10.00)$ & $64.39 \pm 8.99(11.00)$ & -0.88 & 0.38 \\
\hline Weight (kg) & $62.09 \pm 7.46(31.82)$ & $70.39 \pm 11.23(40.68)$ & -3.37 & $<0.01$ \\
\hline$\%$ Body Fat & $22.48 \pm 3.80(15.60)$ & $25.51 \pm 5.31(30.67)$ & -2.55 & 0.01 \\
\hline Age (years) & $19.60 \pm 1.45(6.00)$ & $19.30 \pm 1.02(3.00)$ & 0.93 & 0.36 \\
\hline Sit and Reach Test $(\mathrm{cm})$ & $63.06 \pm 5.64(24.00)$ & $51.70 \pm 8.41(30.67)$ & 6.14 & $<0.01$ \\
\hline $\begin{array}{l}\text { Curl Up Test (number } \\
\text { completed) }\end{array}$ & $77.97 \pm 26.21(69.00)$ & $68.57 \pm 31.08(88.00)$ & 1.27 & 0.21 \\
\hline $\begin{array}{l}\text { Relative Chest Press (lbs. } \\
\text { lifted/ body weight in lbs.) }\end{array}$ & $0.56 \pm 0.11(0.43)$ & $0.64 \pm 0.11(0.49)$ & -2.62 & 0.01 \\
\hline $\begin{array}{l}\text { Relative Leg Press (lbs. } \\
\text { lifted/ body weight in lbs.) }\end{array}$ & $2.22 \pm 0.43(1.68)$ & $2.21 \pm 0.26(1.02)$ & 0.13 & 0.90 \\
\hline $\begin{array}{l}\text { Estimated } \mathrm{VO}_{2 \max } \\
(\mathrm{ml} / \mathrm{kg} / \mathrm{min})\end{array}$ & $43.41 \pm 4.97(28.59)$ & $42.09 \pm 2.98(10.33)$ & 1.25 & 0.22 \\
\hline
\end{tabular}

Table 1. Physical fitness means, standard deviation, ranges, and independent two-tailed $t$-test results for each variable. Bold $p$-values values indicate statistical significance. 
The groups displayed statistically significant differences in three of the six fitness tests. There was a significant difference between groups in body fat percentage measurement, the sit and reach test, and the relative chest press. There was no significant difference between groups in the measurement of relative leg press, $\mathrm{VO}_{2 \max }$ estimate, or the curl up test. The alpha value was set at 0.05 for every test. The mean age for dancer sample was 19.6 years; the average age of the traditional athletes was 19.3 years. The means, variances, and two-tailed independent $t$-test results for each test for the respective groups are displayed in Table 1.

The dancers had a lower percent body fat measurement $(M=22.48 \%, S D=3.8)$ than the traditional athletes $(M=25.51 \%, S D$ $=5.31$ ). The difference was significant $t(58)=-2.55, p=.01$.

The mean scores of the sit and reach test were also significantly different between the groups, with a mean for the dancers of $63.06 \mathrm{~cm}(S D=5.64 \mathrm{~cm})$ and a mean for the traditional athletes of $51.70 \mathrm{~cm}(S D=8.41 \mathrm{~cm}), t(58)=6.14, p<.001$.

The relative chest press was the third test in which a $t$-test revealed a significant difference between the two samples $(t(58)=-2.62$, $p=.01)$. The dancers had a mean relative chest press of $0.56(S D= \pm 0.11)$. The traditional athletes had a higher average relative chest press of $0.64(S D= \pm 0.11)$.

\section{DISCUSSION}

The current study compared the physical demands of collegiate dance to traditional intermittent collegiate sports. It was hypothesized that dancers trained in several styles would display similar fitness to traditional athletes. This was found to be correct for three of the six physical fitness components. Not surprisingly, the dancers showed significantly greater flexibility and lower percent body fat, and the volleyball and softball players displayed greater relative upper body strength. The core strength, relative lower body strength, and estimated aerobic capacity were similar between the softball and volleyball group and the dancer group. Given the physical demands of dance, the core and lower body strength is not unexpected. However, the estimated aerobic fitness of dancers, despite the intermittent style of dance classes, was surprisingly equivalent to that of traditional athletes.

Of the three fitness components showing significant differences, the sit and reach test showed the greatest disparity between groups. This was expected due to the emphasis in dance on extension and flexibility. The dancers also had a significantly lower percent body fat than the athletes; this is likely a reflection of the aesthetic nature of dance and the emphasis on thinness in professional dance. Dancers who continue on a collegiate level tend to fit this body type and have a very low percent body fat. The relatively small variability in height and weight shown by the sample of dancers supports the notion that it is difficult for individuals outside of this aesthetic to continue in dance beyond a certain level.

The traditional athletes had significantly greater relative upper body strength, which suggests that dancers might benefit from more upper body strength training, especially given the contemporary dance trend towards more acrobatic movements heavily influenced by martial arts. Choreography includes more lifts for females to perform, instead of the traditional male and female partnering of classical ballet. Increased upper body strength of dancers would aid the dancer in performing new, more daring dance movements and partnering skills.

There was, no significant difference in lower body or core strength between the two groups. The study utilized relative strength for each muscular strength comparison. When comparing relative strength, differences in body size between participants did not impact the comparison. The leg press test that was used did not mimic the movements of dance, volleyball, or softball. The leg press test did not assess muscular power. The study was limited by available equipment; future studies are needed to evaluate muscular power utilizing movements more specific to dancers and volleyball and softball players. In addition, many participants exceeded the one hundred curl up maximum that was set prior to beginning testing. Continuing the curl up test to exhaustion would provide a more accurate comparison of core strength between testing groups.

The measurement of estimated $\mathrm{VO}_{2 \max }$ was also not significantly different between groups. Previous studies had shown disparities in aerobic gains between styles of dance, so this study tested dancers who are trained in a variety of styles. ${ }^{1,2,5}$ The mean estimated $\mathrm{VO}_{2 \max }$ of the dancers was higher than that of the athletes but the set of dancers had nearly three times the variance, which may reflect the wide variability in aerobic nature between different styles of dance. The mean estimated $\mathrm{VO}_{2 \mathrm{max}}$ of both dancers and traditional athletes was in the $70^{\text {th }}$ percentile for women as determined by the Aerobics Center Longitudinal Study (1970-2002).

Previous studies did not distinguish between types of sport when comparing the aerobic fitness of athletes to dancers. Certain sports require sustained performance while others require short bursts of high intensity effort; dance is more like the latter. By finding similar aerobic fitness in dancers and in softball and volleyball players, it can be suggested that collegiate level dance induces fitness gains comparable to traditional collegiate athletics. 
There were several limitations. All testing was done at a small Midwestern University, so the sample groups were relatively small. Future studies will ideally utilize larger sample groups. In addition, future studies should test dancers in a variety of programs to provide a more comprehensive assessment of dancer fitness, as well as to compare dancers and other traditional sports. Given previous research on the variability of fitness gains between dance genres, the sample of dancers tested may not be representative of dancers at other universities.

Dancers who focus solely on one style of dance would not experience the fitness gains provided by other styles. However, most modern dancers must be well rounded in order to be employable. Dance companies perform a more varied repertoire and require the dancers to be capable of performing a wide variety of styles.

Another limitation of the current study was the use of an estimated $\mathrm{VO}_{2 m a x}$; a metabolic cart could be used to provide a more accurate assessment of aerobic capacity. In addition, body composition could be assessed using more accurate techniques than a BIA. Future research that expands upon these results is necessary to better understand the athletic nature of dance.

In conclusion, there appear to be similar fitness levels between collegiate dancers and traditional athletes, supporting the view of the dancer as a collegiate athlete. This conclusion has several practical implications. One practical result would be inclusion of recreational dance classes as physical education credits. Given that the fitness gains of dance are comparable to those of traditional intermittent sports, it is logical that dance classes would be sufficient to meet physical education requirements, in the same way that schools offer recreational classes in traditional sports.

Another would be an increased number of physicians and trainers on staff with collegiate dance programs and companies. The collegiate sports teams have medical personnel who travel with the team and work with the athletes on a regular basis, yet there are few medical personnel on staff with dance programs at the same universities. Many times dancers are either not treated or must visit an outside physician who is unfamiliar with the dancer and the physical demands of dance. Athletic training education could be expanded to provide greater understanding of the injuries common to dancers.

Despite the aesthetic nature of dance, the current study suggests that collegiate dance provides training adaptations equivalent to traditional intermittent collegiate sports and that dancers ought to be considered college athletes.

\section{REFERENCES}

1. Fromel, K., Vasendova, J., Stratton, G., and Pangrazi, R. P. (2002). Dance as a Fitness Activity: The Impact of Teaching Style and Dance Form. Journal of Physical Education, Recreation \& Dance, 73(5), 26-30, 54.

2. Angioi M., Metsios, G., Koutedakis, V., and Wyon, M. A. (2009). Fitness in Contemporary Dance: A Systematic Review. International Journal of Sports Medicine, 30(7), 475-484. doi:10.1055/s-0029-1202821

3. Flores, R. (1995). Dance for Health: Improving Fitness in African American and Hispanic Adolescents. Public Health Reports (Washington, D.C.: 1974), 110(2), 189-93.

4. Leelarungrayub, D., Saidee, K., Pothongsunun, P., Pratanaphon, S., YanKai, A., and Bloomer, R.J. (2011). Six Weeks of Aerobic Dance Exercise Improves Blood Oxidative Stress Status and Increases Interleukin-2 in Previously Sedentary Women. Journal of Bodywork and Therapies, 15(3), 355-62.

5. Bronner, S., Ojofeitimi, S., Lora, J. B., Southwick, H., Kulak, M. C., Ganboa, J., Rooney, M., Gilman, G., and Gibbs, R. (2014). A Preseason Cardiorespiratory Profile of Dancers in Nine Professional Ballet and Modern Companies. Journal of Dance Medicine and Science, 18(2), 74-85.

6. Grove, J. R., Main, L.C., and Sharp, L. (2013). Stressors, Recovery Processes and Manifestation of Training Distress in Dance. Journal of Dance Medicine and Science, 17(2), 70-78.

7. Koutedakis, Y., and Jamurtas, A. (2004). The Dancer as a Performing Athlete: Physiological Considerations. Sports Medicine, 34(10), 651-661. Retrieved from http://core.ac.uk/ download/pdf/1931746.pdf

8. Ambegaonkar, J. P., Caswell, S. V., Winchester, J. B., Caswell, A. A., and Andre, M.J. (2012). Upper-body Muscular Endurance in Female University-level Modern Dancer: A Pilot Study. Journal of Dance Medicine and Science, 16(1), 3-7.

9. Rafferty, S. (2010). Considerations for Integrating Fitness into Dance Training. Journal of Dance Medicine and Science, 14(2), 45-49.

10. Angioi, M., Metsios, G., Twitchett, E., Koutedakis, Y., and Wyon, M. (2009). Association Between Selected Physical Fitness

Parameters and Aesthetic Competence in Contemporary Dancers. Journal of Dance Medicine and Science, 13(4), $115-123$. 


\section{ABOUT THE STUDENT AUTHOR}

Leanna Miller graduated from Anderson University, Indiana in Spring 2016 with Bachelor's Degrees in Exercise Science and Psychology and a minor in Dance Performance. Leanna was an honors student, as well as a member of the Center for Public Service, Psi Chi, Alpha Chi, and Phi Epsilon Kappa. Leanna is interested in health and in the use of non-traditional therapeutic modalities in rehabilitation, as well as treatment of and injury prevention for dancers. Leanna plans to pursue a career in geriatric rehabilitation, using her dance background in conjunction with her exercise science and psychology education to best serve the aging population.

\section{PRESS SUMMARY}

Research indicates that dance can provide many benefits in physical fitness, yet the social and medical treatment of dancers differs vastly from that of athletes. This study tested six different physical fitness components of dancers and traditional athletes to provide a direct comparison of the gains in fitness from dance and traditional sports (softball and volleyball). This study found that although there was some variability, overall fitness of dancers seems to be equivalent to that of athletes, supporting the application of sports medicine to the field of dance. 